\title{
Efficacy of thermoablation in treating cervical precancerous lesions in a low-resource setting
}

This article was published in the following Dove Press journal:

International Journal of Women's Health

\section{Phuong Lien Tran' \\ Bruno Kenfack ${ }^{2}$ \\ Eveline Tincho Foguem ${ }^{3}$ \\ Manuela Viviano' \\ Liliane Temogne ${ }^{2}$ \\ Pierre-Marie Tebeu ${ }^{4}$ \\ Rosa Catarino' \\ Anne-Caroline Benski' \\ Pierre Vassilakos ${ }^{5}$ \\ Patrick Petignat ${ }^{\prime}$}

'Division of Gynecology, Department of Gynecology and Obstetrics,

Geneva University Hospitals,

Geneva, Switzerland; '² Department of Biomedical Sciences, University of Dschang, Dschang, Cameroon; ${ }^{3}$ Department of Gynecology and Obstetrics, District Hospital of Biyem-Assi, Yaoundé, Cameroon; ${ }^{4}$ Department of Gynecology and Obstetrics, University Centre Hospital, Yaoundé, Cameroon; ${ }^{5}$ Geneva Foundation for Medical Education and Research, Geneva, Switzerland
Correspondence: Phuong Lien Tran Division of Gynecology, Department of Gynecology and Obstetrics, Geneva University Hospitals, 30 Boulevard de la Cluse, Geneva 1205, Switzerland

Tel +4l 223724432

Fax +4I 223724 I88

Email phuong_lien_tran@yahoo.com
Introduction: Treating cervical intraepithelial neoplasia (CIN) grades 2 and 3 is the recommended strategy for preventing invasive carcinoma in low- and middle-income countries (LMICs). Our objective was to assess the efficacy of thermoablation in the treatment of CIN2 and CIN3 in a screen-and-treat approach.

Methods: Women aged 30-49 years in Dschang, Cameroon, were invited to undergo vaginal sampling for human papillomavirus (HPV), samples being assessed by an Xpert HPV Assay. HPV-positive women underwent visual inspection with acetic acid (VIA) and visual inspection with Lugol's iodine (VILI), cervical biopsy, and endocervical curettage. Women positive for HPV-16/18/45 or other HPV types with abnormal VIA/VILI were treated by thermoablation on the same day. The primary outcome was persistence of high-grade disease on cytologic examination at 12 months.

Results: Of a total of 1,012 recruited women, 188 were HPV-positive, 121 patients required thermoablation, and 99 had a CIN of grade $<2$, making the overtreatment rate $9.9 \%$. The cure rate for CIN2 and CIN3 at 12 months was $70.6 \%$. Failure (higher risk of persistent disease) was associated with the presence of occult endocervical lesions at baseline diagnosis (adjusted odds ratio $[\mathrm{aOR}]=128.97$ [95\% confidence interval [CI], 8.80-1,890.95]; $p<0.0001$ ). First sexual intercourse before the age of 15 was also a risk factor ( $\mathrm{aOR}=0.003$ [ $95 \% \mathrm{CI}, 0.001-0.61] ; p=0.023$ ).

Conclusion: In LMICs, use of thermoablation in a screen-and-treat approach is a valuable treatment option for CIN2 and CIN3. Studies comparing thermoablation with cryotherapy are needed to determine the most appropriate treatment for cervical precancer in such countries.

Keywords: cervical cancer, HPV testing, self-sampling, thermoablation

\section{Introduction}

Cervical cancer (CC) is the fourth most common and lethal cancer in women worldwide. ${ }^{1}$ Almost $85 \%$ of CC occurs in low- and middle-income countries (LMICs), mainly because of the lack of efficient primary and secondary prevention programs. ${ }^{2}$ Effective treatment of precancerous lesions through screening has the potential to drastically reduce $\mathrm{CC}$ incidence.

The World Health Organization (WHO) ${ }^{3}$ recommends a screen-and-treat approach comprising primary HPV screening followed by visual inspection triage after the application of acetic acid and Lugol's iodine (VIA/VILI) and then treatment, if needed, as the optimal CC prevention strategy in LMICs. This strategy optimizes physician availability as well as minimizes loss to follow-up. ${ }^{4}$

Conservative ablative methods are more established in LMICs because they are easy to implement and are associated with low morbidity. The adoption of a screenand-treat strategy using cryotherapy has been demonstrated to achieve high cure rates ( $88 \%$ for cervical intraepithelial neoplasia [CIN] of grades $1-2$, and $70 \%$ for CIN3) 
in LMICs. ${ }^{5}$ Cryotherapy is easy to administer and can be effectively performed by mid-level surgeons. ${ }^{6}$ However, the procedure poses certain challenges, such as the need for continuous refills of refrigerant gas, bulky equipment that limits mobility in the field, and problems in system connectivity, all of which impede its sustainable use in LMIC.

The cure rates of thermoablation and cryotherapy are similar. ${ }^{7}$ Because thermoablation uses an electric battery to generate temperatures of $100^{\circ} \mathrm{C}-120^{\circ} \mathrm{C}$ for ablation of cervical lesions, it requires no consumables and, therefore, provides a feasible option in LMICs. This ablative treatment technique is indicated in nonpregnant women of any age with CIN2 and CIN3, provided the entire transformation zone is visible and there is no suspicion of endocervical involvement or invasive disease. The procedure is quick and achieves a treatment depth of 4-7 mm. ${ }^{8}$ The equipment is light, easily portable, and has an incorporated automatic self-sterilization feature. In addition, only few adverse effects have been reported. ${ }^{8}$

Nevertheless, thermoablation was not included in the recent updated WHO guidelines for screening and treatment of precancerous lesions for $\mathrm{CC}$ prevention ${ }^{3}$ because it is used infrequently worldwide and limited information about its use in LMICs is available. ${ }^{7}$

The aim of the present study was to determine the 12-month effectiveness of thermoablation in the treatment of precancerous cervical lesions in a low-resource setting.

\section{Methods}

\section{Study setting}

The Faculty of Medicine and Biomedical Sciences (Yaoundé, Cameroon), University of Dschang (Cameroon), and Geneva University Hospitals have been working together to evaluate innovative CC screening options with the aim of developing a CC screening approach adapted to the needs and means of women in Cameroon.

\section{Study participants and procedure}

The study site, a geographically well-defined area in West Region, Cameroon, has an estimated population of 250,000 .

Women were recruited through announcements in women's associations, churches, and integrated health centers in Dschang Health District (chief nurses of each center were responsible for recruitment); announcements were played on local radio stations, and banners were displayed in hospitals and other public places such as markets to inform women of the screening campaign. These specified eligibility criteria and the duration and location of screening. The focus was on this campaign being free to participants. Women in the District of Dschang were invited, and the study was conducted in collaboration with the University of Dschang.

Criteria for inclusion in the study included age 30-49 years and compliance with the study protocol. Exclusion criteria included pregnancy, history of cervical therapy for CIN, or total hysterectomy. All eligible women were asked to sign an informed consent form before taking part in the study. Ethical approval was obtained from the National Ethics Committee of Cameroon (2015/02/559/CE/CNERSH/SP) and from the Ethical Cantonal Board of Geneva, Switzerland (CCER 15-068).

During a screening campaign from July to December 2015, health care providers instructed all participants to perform human papillomavirus (HPV) self-sampling using a dry cotton swab. The self-collected vaginal samples were analyzed using a HPV GeneXpert (GeneXpert ${ }^{\mathbb{R}}$ IV; Cepheid, Sunnyvale, CA, USA). This assay performs a real-time polymerase chain reaction that allows simultaneous detection of 14 high-risk (HR) HPV types. The results (positivity/ negativity) were classified into three categories - HPV 16, HPV 18/45, and 11 other HR-HPV types (31, 33, 35, 39, 51, $52,56,58,59,66,68)$ - and were made available within an hour. This point-of-care test enabled screening, triage, and treatment of women on the same day. Women who tested positive for HR-HPV genotypes were invited to undergo a gynecological examination with VIA/VILI. The standard diagnostic method for the disease is histological evaluation; thus, a cervical biopsy was obtained, and an endocervical curettage (ECC) was performed on all HPV-positive women as a quality control. Biopsies were performed in the area of visible pathology or in the transformation zone at 6 o'clock when no lesion was visible. ECC results were collected separately from the biopsy.

Women who were either "HPV 16/18/45-positive" or "positive to other HR-HPV types and to VIA" were considered screen-positive and therefore require treatment. Screenpositive women were considered eligible for thermoablation if: (1) the lesion extended $<2 \mathrm{~mm}$ into the endocervical canal; (2) the anatomy of the cervix allowed treatment of the entire lesion (multiple applications were permitted); (3) the lesion did not extend onto the vaginal wall; and (4) there was no clinical suspicion of cancer. No anesthetic was administered prior to the procedure. Treatment was performed using a thermocoagulator (WISAP ${ }^{\circledR}$; Medical Technology GmbH, Brunnthal/Hofolding, Germany) after delineation of the lesion using Lugol's iodine. Thermoablation was achieved using a probe that was heated to $100^{\circ} \mathrm{C}$ and then applied to 
the cervix for 60 seconds. If the entire abnormal area required to be treated, this process was repeated. After use, the probe was washed using cold water, dried, and then heated for about 45 seconds at $120^{\circ} \mathrm{C}$ to sterilize it. ${ }^{9}$

\section{Outcome measures and follow-up}

Screen-positive women were invited for 6- and 12-month follow-up visits, which included HPV self-sampling, HPV sampling performed by a physician, pelvic examination with VIA/VILI, and cervical cytology. Cervical specimens were collected using a broom-like device and fixed in BD SurePath ${ }^{\mathrm{TM}}$ liquid medium (Becton Dickinson, Franklin Lakes, NJ, USA). Biopsies, followed by ECC, were performed on all patients with a positive VIA/VILI test, history of CIN2, or more severe lesions (CIN2+). ECC results were collected separately from biopsy. The slices were read by an expert anatomopathologist in Cameroon, with a random quality control by another expert anatomopathologist in Geneva, Switzerland.

The primary outcome in the present study was the absence of CIN2+ disease at the 12-month follow-up, being defined as absence of either CIN2 and CIN3 in a biopsy or a highgrade squamous intraepithelial lesion (HSIL) on cytologic examination.

A woman's disease status was classified as negative if ASCUS (atypical squamous cells of undetermined significance) and nonpathological VIA/VILI were identified, because the probability of diagnosing CIN2+ after an ASCUS-positive smear is $<10 \%,{ }^{10}$ whereas it was classified as positive if ASC-H (atypical squamous cells suspicious of high-grade lesion) were identified; these women were considered to have high-grade cervical lesions.

Cure rate was defined as the percentage of women with no evidence of persistent disease at 12 months after a baseline diagnosis of CIN2 or CIN3. Overtreatment was defined as treatment of women who either had no lesion or had lesions suggesting CIN of grade $<2$ on baseline screening of cervical biopsies. Patients were informed about the risk of overtreatment and the possible adverse effects of thermoablation, though the latter are considered minimal.

\section{Data analysis and statistics}

Data were analyzed with a 2013 StataCorp software package (release 13; Stata Statistical Software, College Station, TX, USA). Quantitative variables are expressed as means and standard deviations, and qualitative variables as percentages, unless otherwise stated. Categorical variables were analyzed with Pearson's $\chi^{2}$ or Fisher's test and continuous and ordinal variables with the Mann-Whitney $U$-test or the Kruskal-Wallis test, as appropriate. The prognostic value of each study variable for overall histological persistence/ recurrence was first assessed with univariate Cox regression analysis. Variables were then included in the multivariate Cox proportional hazard model in a stepwise method. All reported $p$-values are two-tailed. $p<0.05$ was considered to denote statistical significance.

\section{Results \\ Main results}

The primary screening campaign in Dschang recruited 1,012 eligible women, of whom $188(18.6 \%)$ were HR-HPVpositive. Of these 188 women, 121 (64.3\%) were positive on screening for either HPV-16/18/45 or other HPV, and VIA/ VILI-positive, and were therefore eligible for treatment by thermoablation. All 121 screen-positive women had a VIA/ VILI assessment to exclude obvious CC, and none were found to have lesions suspicious of cancer. Median time to the first follow-up visit, for which 130 women presented, was 8.4 months, and to the second follow-up visit, for which 112 women presented, was 14.5 months. One patient with CIN2 and CIN3 did not attend her 6- and 12-month follow-up visits, and another attended the 6th month, but not the 12 th month follow-up visit.

There was a 97.1\% agreement between cytology and histology findings in women for whom biopsy results were available. Thus, cytology was considered an accurate means of ascertaining disease status.

Figure 1 summarizes the management of participants in the study. The characteristics of HPV-positive participants are reported in Table 1.

\section{Disease status at baseline screening}

Of the 121 treated women, 92 (76.1\%) had negative biopsies and ECC at baseline screening, whereas seven (5.8\%) were classified as having CIN1 disease. One woman with a CC that was not identified at the clinical assessment was inadvertently treated by thermoablation. This patient was recalled for definitive surgery when her biopsy result was obtained. Her final diagnosis was FIGO (International Federation of Gynecology and Obstetrics) stage Ib1 squamous cell carcinoma. Although these decisions to treat lesions suggestive of CIN of grade $<2$ were not justified, the rate of overtreatment was only $9.9 \%(100 / 1,012$ patients screened). Of the 100 overtreated patients, $13 \%$ were HPV16-positive, 32\% HPV18/45, and the remaining $55 \%$ other HPV-positive. Because we used a screen-and-treat 


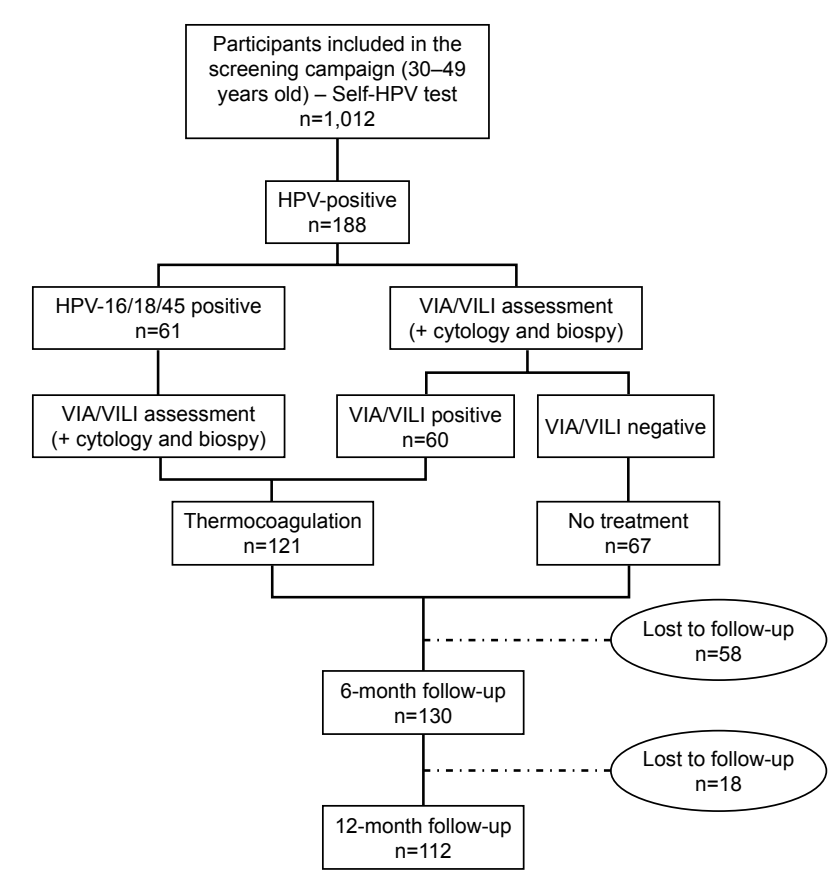

Figure I Flowchart of study protocol.

Abbreviations: HPV, human papillomavirus; VIA, visual inspection with acetic acid; VILI, visual inspection with Lugol's iodine.

approach, the results of biopsy and ECC samples were not available at the time of treatment. Subsequent histological examination revealed the presence of CIN2 in two women who had received no treatment initially; these women were recalled for appropriate therapy.

\section{Disease status at 6 and 12 months}

Of the treated women, $88.7 \%$ had no evidence of disease after 6 months, and $86.7 \%$ after 12 months. However, $76.0 \%$ of treated patients had had no evidence of disease before thermoablation. Of the 17 patients with a baseline diagnosis of CIN2+ disease (three CIN2, 14 CIN3, and 10 of them having a positive ECC), examination of Pap smears obtained at the 6th month follow-up showed that seven patients had persistent HSIL, as did five at the 12th month. Thus, for the 17 patients with CIN2+ disease who underwent thermoablation, the cure rate was $58.8 \%(10 / 17)$ and $70.6 \%(12 / 17)$ at 6 and 12 months, respectively (Figure 2).

\section{Factors associated with treatment failure at 12 months}

Failure was partially attributable to the presence of occult endocervical lesions. Women identified with endocervical involvement with CIN2 and CIN3 at baseline (discovered on histological examination several weeks after treatment) were associated with a much higher risk of abnormal
Table I Sociodemographic and clinical characteristics of study participants $(n=188)$

\begin{tabular}{|c|c|c|}
\hline Variable & $\begin{array}{l}\text { Treated } \\
\text { patients } \\
\mathrm{n}=\mid \mathbf{2} \text { I, n (\%) }\end{array}$ & $\begin{array}{l}\text { Nontreated } \\
\text { patients } \\
\text { n=67, n (\%) }\end{array}$ \\
\hline Age (years), mean $\pm S D$ & $38.7 \pm 5.3$ & $38.6 \pm 6.1$ \\
\hline $\begin{array}{l}\text { Age at first sexual intercourse (years), } \\
\text { mean } \pm S D\end{array}$ & $18.0 \pm 2.8$ & $17.9 \pm 2.6$ \\
\hline Gestity, mean \pm SD & $4.9 \pm 2.2$ & $5.0 \pm 2.4$ \\
\hline Parity, mean \pm SD & $4.0 \pm 1.8$ & $4.2 \pm 2.1$ \\
\hline Number of sexual partners, mean $\pm S D$ & $4.2 \pm 3.0$ & $3.6 \pm 2.5$ \\
\hline Lost to follow-up at 12 months & $3 \mathrm{I}(25.6)$ & $19(28.4)$ \\
\hline \multicolumn{3}{|l|}{ Marital status } \\
\hline Single & $8(6.6)$ & $5(7.5)$ \\
\hline With a partner & II $3(93.4)$ & $62(92.5)$ \\
\hline \multicolumn{3}{|l|}{ Education level } \\
\hline None & $0(0)$ & $\mathrm{I}(\mathrm{I} .5)$ \\
\hline Elementary school & $27(22.3)$ & $12(17.9)$ \\
\hline Apprenticeship & $3(2.5)$ & $0(0)$ \\
\hline High school & $72(59.5)$ & $44(65.7)$ \\
\hline University & $19(15.7)$ & $8(11.9)$ \\
\hline Other & $0(0)$ & $2(3)$ \\
\hline \multicolumn{3}{|l|}{ Employment status } \\
\hline Employed & $80(66.1)$ & $4 \mid(6 I .2)$ \\
\hline Farmer & $7(5.8)$ & $5(7.5)$ \\
\hline Housewife & $29(24.0)$ & $19(28.3)$ \\
\hline Other & $5(4.1)$ & $2(3)$ \\
\hline \multicolumn{3}{|l|}{ Contraception } \\
\hline None & $92(76.0)$ & $49(73.1)$ \\
\hline Pill & $2(1.7)$ & $\mathrm{I}(\mathrm{I} .5)$ \\
\hline IUD & $3(2.5)$ & I (I.5) \\
\hline Injection & $6(5.0)$ & $4(6)$ \\
\hline Condom & $12(9.9)$ & $9(13.4)$ \\
\hline Other & $6(4.9)$ & $3(4.5)$ \\
\hline \multicolumn{3}{|l|}{ HPV test result at baseline screening* } \\
\hline HPV-I6 & $18(14.9)$ & $0(0)$ \\
\hline HPV-I8/45 & $27(22.3)$ & $0(0)$ \\
\hline Other HR-HPV & 61 (50.4) & $64(95.5)$ \\
\hline HPV-16+ other HPV & $2(1.7)$ & $0(0)$ \\
\hline HPV-I8/45+ other HPV & $13(10.7)$ & $0(0)$ \\
\hline \multicolumn{3}{|l|}{ Diagnosis } \\
\hline Negative & $92(76.1)$ & $58(86.5)$ \\
\hline $\mathrm{CINI}$ & $7(5.8)$ & $3(4.5)$ \\
\hline $\mathrm{CIN} 2$ & $3(2.5)$ & $0(0)$ \\
\hline CIN3 & $13(10.7)$ & $2(3)$ \\
\hline Invasive carcinoma & $\mathrm{I}(0.8)$ & $0(0)$ \\
\hline Invalid/no biopsy & $5(4.1)$ & $4(6)$ \\
\hline Endocervical involvement & $10(8.3)$ & I (I.5) \\
\hline
\end{tabular}

Note: *HPV self-sample test results.

Abbreviations: CIN I/2/3, cervical intraepithelial neoplasia of grades $1 / 2 / 3$; HPV, human papillomavirus; HR, high risk; IUD, intrauterine device; SD, standard deviation.

cytology at the 12th month follow-up compared with women without identified endocervical involvement (adjusted odds ratio $[\mathrm{aOR}]=128.97$ [95\% confidence interval $[\mathrm{CI}]$, 8.80-1,890.95]; $p<0.0001)$. When patients with microscopic endocervical lesions identified at ECC were excluded, the 


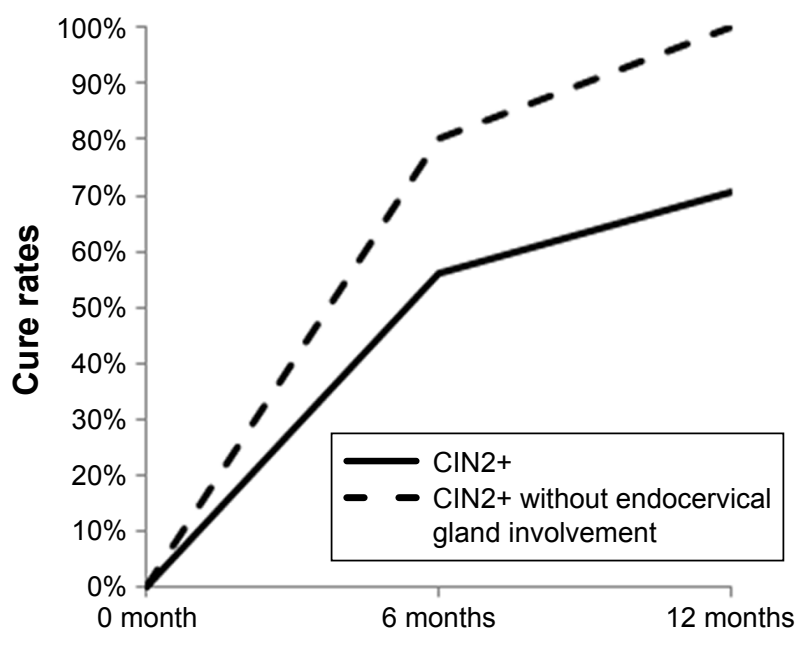

Figure 2 Cure rates after thermoablation according to baseline diagnosis. Abbreviation: CIN2+, cervical intraepithelial neoplasia of grade 2 or more.

cure rate was $100 \%$ at 12 months (Figure 2). A forest plot of thermoablation versus other treatment methods is presented in Figure 3. Moreover, women with first sexual intercourse before the age of 15 had a higher risk of persistent disease than those who became sexually active later in life
$(\mathrm{aOR}=0.003$ [95\% CI, 0.001-0.61]; $p=0.023)$. However, age, number of sexual partners, educational level, marital status, employment status, contraception use, and HPV test results at baseline screening were not associated with abnormal cytology (Table 2).

\section{Discussion}

Thermoablation appears to be a feasible screen-and-treat approach in LMIC. It is an effective means of treating women with precancerous cervical lesions and may reduce the risk of CC ${ }^{8}$ In the present study, the thermoablation cure rate was $70.6 \%$, which is comparable with the rate achieved by cryotherapy in LMICs, which is reportedly $70 \%$ for CIN3. ${ }^{5}$ Our results showed a little inferior overall efficacy of thermoablation of $94.0 \%(95 \%$ CI, 91\%-96\%) reported in Dolman's meta-analysis, ${ }^{8}$ however, these results are difficult to extrapolate to LMICs as there were only three studies in that review that employed a screen-and-treat strategy and only one of them was conducted in an LMIC (India).

To date, there are few published data on effective delivery of thermoablation in screening programs in LMICs.

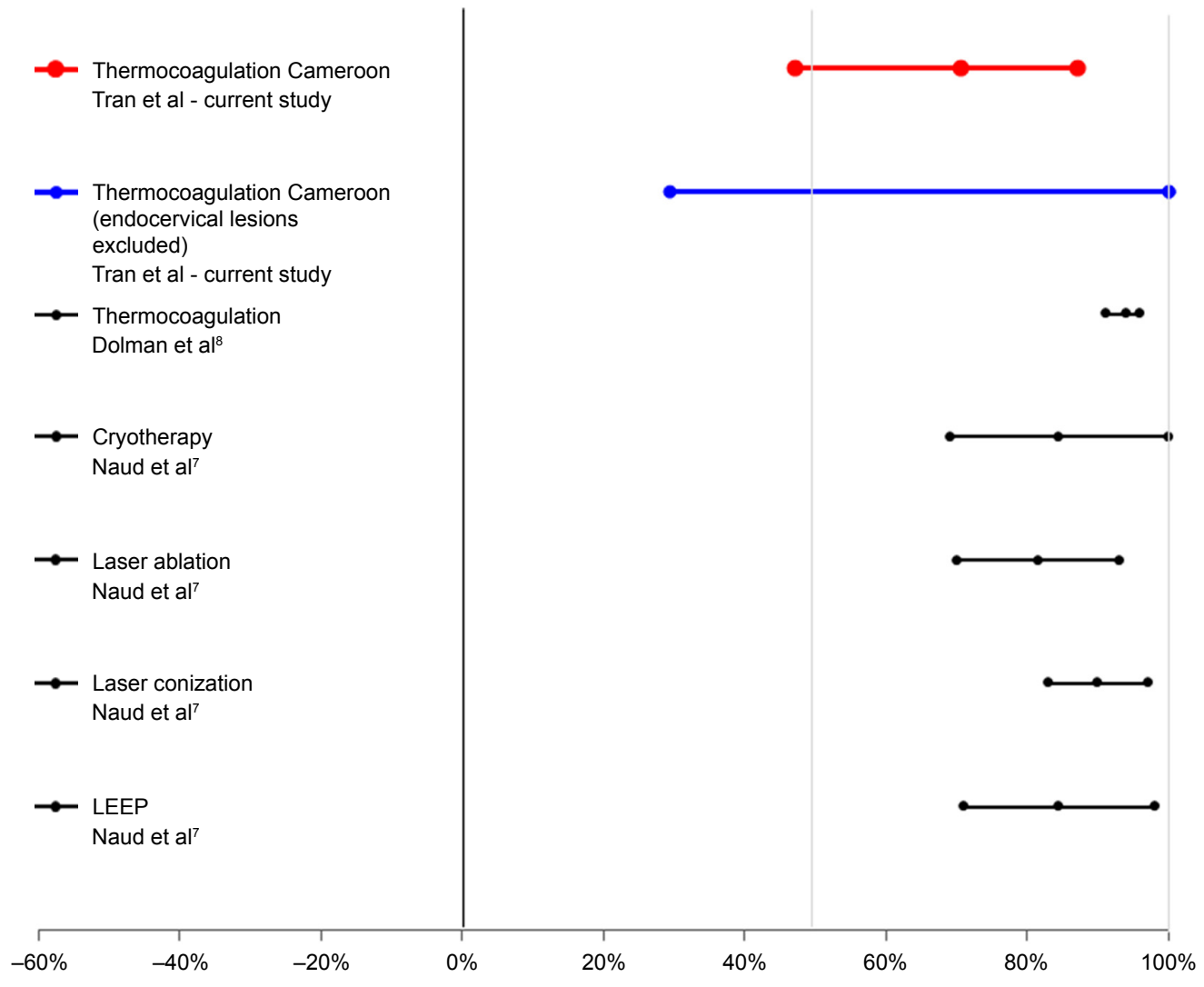

Figure 3 Forest plot comparing the efficacy of thermoablation with that of other treatments.

Note: Mid-points represent the mean efficacy of each treatment, and the width of the line shows $95 \%$ confidence interval.

Abbreviation: LEEP, loop electrosurgical excision procedure. 
Table 2 Univariate and multivariate analyses of factors associated with persistent HSIL

\begin{tabular}{|c|c|c|c|c|c|c|}
\hline \multirow[t]{2}{*}{ Variables } & \multicolumn{3}{|c|}{ Univariate } & \multicolumn{3}{|c|}{ Multivariate } \\
\hline & OR & $95 \% \mathrm{Cl}$ & $P$-value & aOR & $95 \% \mathrm{Cl}$ & $P$-value \\
\hline \multicolumn{7}{|l|}{ Age (years) } \\
\hline $30-40$ & I (ref) & & & & & \\
\hline $4 I-50$ & 1.41 & $0.19-10.52$ & 0.737 & & & \\
\hline \multicolumn{7}{|c|}{ Age at first sexual intercourse (years) } \\
\hline$<15$ & I (ref) & & & I (ref) & & \\
\hline$\geq 15$ & 0.03 & $0.002-0.28$ & $0.003 *$ & 0.003 & $0.00 \mathrm{I}-0.6 \mathrm{I}$ & $0.023 *$ \\
\hline \multicolumn{7}{|l|}{ Parity } \\
\hline$<4$ & I (ref) & & & & & \\
\hline$\geq 4$ & 1.56 & $0.15-15.65$ & 0.708 & & & \\
\hline \multicolumn{7}{|c|}{ Number of sexual partners } \\
\hline$<4$ & I (ref) & & & I (ref) & & \\
\hline$\geq 4$ & 6.16 & $0.70-54.27$ & 0.101 & 5.7 & $0.20-161.18$ & 0.307 \\
\hline \multicolumn{7}{|l|}{ Marital status } \\
\hline Single & I (ref) & & & I (ref) & & \\
\hline With a partner & 0.15 & $0.01-1.83$ & 0.138 & 0.12 & $0.003-4.06$ & 0.236 \\
\hline \multicolumn{7}{|l|}{ Education level } \\
\hline$<$ High school & I (ref) & & & & & \\
\hline$\geq$ High school & 0.28 & $0.04-2.14$ & 0.22 & & & \\
\hline \multicolumn{7}{|l|}{ Employment status } \\
\hline Housewife & I (ref) & & & & & \\
\hline Employed-Farmer & 1.24 & $0.12-12.54$ & 0.855 & & & \\
\hline \multicolumn{7}{|l|}{ Contraception } \\
\hline No & I (ref) & & & & & \\
\hline Yes & 1.11 & $0.11-11.25$ & 0.933 & & & \\
\hline \multicolumn{7}{|l|}{ Endocervical invasion } \\
\hline No & I (ref) & & & & & \\
\hline Yes & 1.23 & $|3.65-|, 108.33$ & $<0.0001$ & 128.97 & $8.80-1,890.95$ & $<0.000 I^{*}$ \\
\hline \multicolumn{7}{|c|}{ HPV test result at baseline screening } \\
\hline HPV-I6 & I (ref) & & & & & \\
\hline HPV-I8/45 & 0.74 & $0.06-8.77$ & 0.808 & & & \\
\hline Other HR-HPV & 0.31 & $0.03-3.63$ & 0.352 & & & \\
\hline
\end{tabular}

Note: *Statistical significance (bold values).

Abbreviations: aOR, adjusted odds ratio; Cl, confidence interval; HPV, human papillomavirus; HSIL, high-grade squamous intraepithelial lesion; OR, odds ratio; ref, reference.

Among the studies published in India, ${ }^{9}$ Malawi, ${ }^{11}$ and Nigeria, ${ }^{12}$ the cure rates varied between $80 \%$ and $93.3 \%$; however, in two of those studies, the outcome was recurrence of VIA positivity without confirmation by the gold standards of cytology or histology. Indeed, despite its sensitivity of $50 \%-96 \%$ for detection of CIN2+ in primary screening, the true value of VIA alone in a follow-up context is not well established and may be lower in terms of sensitivity than in primary screening. ${ }^{13,14}$

Overtreatment is a major concern with a screen-and-treat approach for CC prevention because a large proportion of screen-positive women treated were subsequently found not to have a premalignant cervical lesion. ${ }^{15,16}$ Reported overtreatment rates vary between $12.5 \%$ and $45 \%{ }^{11,17}$ and are similar to those reported for screen-and-treat strategies using cryotherapy. ${ }^{11}$ The high proportion of overtreatment may be attributable to the low positive predictive values of visual methods, which is around 52\%. ${ }^{18}$ Histological findings of VIA-directed biopsies do not correlate well with screening findings, as evidenced by the fact that 92 of 121 women treated in our study who were labeled as screen-positive were subsequently found to have no histological evidence of the disease. On the one hand, by using primary HPV-based screening followed by genotyping or VIA/VILI triage, we achieved lower overtreatment rates than those previously reported. However, on the other hand, using VIA/VILI as triage, we initially undertreated two women who were later recalled for definitive treatment, thanks to having systematically taken biopsies and performed ECC on all screenpositive women.

Patients evaluated 4 weeks after treatment reported only minor adverse events such as vaginal discharge and low pain scores. ${ }^{19}$ In a recent review, ${ }^{8,20}$ other adverse effects, including vaginal bleeding, fainting, cervical stenosis, and 
local cervical infection, were reported, though rarely $(<1 \%)$. The most frequent adverse effect was pain attributable to uterine cramps; this did not appear to prevent therapy or limit adherence to the study protocol. Moreover, there were no demonstrable adverse effects on fertility or delivery in pregnancies conceived after thermoablation; however, data concerning this are limited. Advantages of a screen-and-treat strategy include that it reduces treatment delay and is easy to implement, thus minimizing loss to follow-up. These characteristics render this approach particularly useful in resource-limited settings, where travel is costly and timeconsuming, discouraging patients from returning to treatment in the absence of symptoms. ${ }^{11} \mathrm{We}$ found that more women who were untreated were lost to follow-up compared to treated women; however, this finding was caused by random fluctuations and not significant.

We found that women who had first sexual intercourse at 15 years of age or later had protection against disease recurrence, whereas the presence of endocervical involvement at baseline was associated with a higher likelihood of treatment failure. The latter, along with multiparity $\geq 2$, has been described previously by Papoutsis et al. ${ }^{21,22}$ Other studies have found an association between increased risk of recurrence of CIN and HIV infection, ${ }^{23}$ CD4 cell count $<200$ cells $/ \mathrm{mm}^{3}$ among HIV-positive women, and age $<30$ years. ${ }^{12}$ Although extension into the endocervical canal was an exclusion criterion, microscopic involvement of the endocervical canal was only identified a posteriori on the results of the ECC. Reasons for treatment failure include inability of the probe's tip to reach the endocervical canal to treat any microscopic lesions inside it. When we excluded patients with endocervical lesions, the cure rate was $100 \%$.

The key strength of this study is the clinical and pathological follow-up incorporated into the study design; this included a pelvic examination with VIA/VILI, biopsy, and cytology for all HPV-positive participants, minimizing the risk of missed disease. Another strength is our screen-andtreat approach performed in a single visit.

This study also has some limitations, including the small sample size and high rate of loss to follow-up, with attending for follow-up being costly and time-consuming for participants.

To the best of our knowledge, the study reported here is one of the largest studies to use thermoablation for the treatment of precancerous cervical lesions in LMICs. The overall cure rate was $70.6 \%$, and $100 \%$ after exclusion of participants with microscopic endocervical involvement. Improvements in treating microscopic regions of the endocervix are still needed. Nonetheless, we consider that the screen-and-treat approach, with its safety, acceptability, rapidity, simplicity of use, and low cost, is a viable option for the management of suitable precancerous cervical lesions.

More studies, including randomized trials comparing thermoablation with cryotherapy, are needed to determine whether thermoablation may become the new gold standard for treatment in this setting.

\section{Acknowledgment}

The authors thank Jovanny Tsuala Fouogue for his critical input of important intellectual content.

\section{Disclosure}

The authors report no conflicts of interest in this work.

\section{References}

1. Ferlay J, Soerjomataram I, Dikshit R, et al. Cancer incidence and mortality worldwide: sources, methods and major patterns in GLOBOCAN 2012. Int J Cancer. 2015;136(5):E359-E386.

2. Screening for cervical cancer. Available from: http://www.uptodate. com/contents/screening-for-cervical-cancer? source=search_result\& search $=$ screening + for + cervical + cancer \&selectedTitle $=1 \% 7 \mathrm{E} 150$. Accessed March 20, 2017

3. World Health Organization. Guidelines for screening and treatment of precancerous lesions for cervical cancer prevention [cited December 29, 2016]. Available from: http://www.who.int/reproductivehealth/ publications/cancers/screening_and_treatment_of_precancerous_ lesions/en/. Accessed March 20, 2017.

4. Ebisch RM, Rovers MM, Bosgraaf RP, et al. Evidence supporting see-and-treat management of cervical intraepithelial neoplasia: a systematic review and meta-analysis. BJOG. 2016;123(1):59-66.

5. Sherris J, Wittet S, Kleine A, et al. Evidence-based, alternative cervical cancer screening approaches in low-resource settings. Int Perspect Sex Reprod Health. 2009;35(3):147-154.

6. Sauvaget C, Muwonge R, Sankaranarayanan R. Meta-analysis of the effectiveness of cryotherapy in the treatment of cervical intraepithelial neoplasia. Int J Gynaecol Obstet. 2013;120(3):218-223.

7. Naud PS, Muwonge R, Passos EP, Magno V, Matos J, Sankaranarayanan R. Efficacy, safety, and acceptability of thermoablation for treatment of cervical intraepithelial neoplasia in a hospital setting in Brazil. Int $J$ Gynaecol Obstet. 2016;133(3):351-354.

8. Dolman L, Sauvaget C, Muwonge R, Sankaranarayanan R. Metaanalysis of the efficacy of cold coagulation as a treatment method for cervical intraepithelial neoplasia: a systematic review. BJOG. 2014; 121(8):929-942.

9. Joshi S, Kulkarni V, Darak T, et al. Cervical cancer screening and treatment of cervical intraepithelial neoplasia in female sex workers using "screen and treat" approach. Int J Womens Health. 2015;7:477-483.

10. Nygård JF, Sauer T, Skjeldestad FE, Skare GB, Thoresen SØ. CIN 2/3 and cervical cancer after an ASCUS pap smear. A 7-year, prospective study of the Norwegian population-based, coordinated screening program. Acta Cytol. 2003;47(6):991-1000.

11. Campbell C, Kafwafwa S, Brown H, et al. Use of thermo-coagulation as an alternative treatment modality in a 'screen-and-treat' programme of cervical screening in rural Malawi. Int J Cancer. 2016;139(4): 908-915.

12. Oga EA, Brown JP, Brown C, et al. Recurrence of cervical intraepithelial lesions after thermo-coagulation in HIV-positive and HIV-negative Nigerian women. BMC Womens Health. 2016;16:25. 
13. Orang'o O, Liu T, Christoffersen-Deb A, et al. Use of VIA, Pap smear, or HR-HPV testing in women living with HIV/AIDS for post-treatment cervical cancer screening: same tests, different priorities. AIDS. Epub 2016 Nov 7.

14. Louwers J, Zaal A, Kocken M, et al. Dynamic spectral imaging colposcopy: higher sensitivity for detection of premalignant cervical lesions. BJOG. 2011;118(3):309-318.

15. Fokom-Domgue J, Vassilakos P, Petignat P. Is screen-and-treat approach suited for screening and management of precancerous cervical lesions in Sub-Saharan Africa? Prev Med. 2014;65:138-140.

16. Arbyn M, Sankaranarayanan R, Muwonge R, et al. Pooled analysis of the accuracy of five cervical cancer screening tests assessed in eleven studies in Africa and India. Int J Cancer. 2008;123(1):153-160.

17. Sankaranarayanan R. 'See-and-treat' works for cervical cancer prevention: what about controlling the high burden in India? Indian J Med Res. 2012;135(5):576-579.

18. Barut MU, Kale A, Kuyumcuoglu U, et al. Analysis of sensitivity, specificity, and positive and negative predictive values of smear and colposcopy in diagnosis of premalignant and malignant cervical lesions. Med Sci Monit. 2015;21:3860-3867.
19. Viviano M, Kenfack B, Catarino R, et al. Feasibility of thermoablation in a screen-and-treat approach for the treatment of cervical precancerous lesions in sub-Saharan Africa. BMC Womens Health. 2017;17(1):2.

20. Zawislak A, Price JH, McClelland HR, Storey RG, Caughley L. Efficacy of cervical intraepithelial neoplasia (CIN) treatment by cold coagulation. Ulster Med J. 2003;72(1):10-15.

21. Jensen KE, Schmiedel S, Norrild B, Frederiksen K, Iftner T, Kjaer SK. Parity as a cofactor for high-grade cervical disease among women with persistent human papillomavirus infection: a 13-year follow-up. $\mathrm{Br} J$ Cancer. 2013;108(1):234-239.

22. Papoutsis D, Underwood M, Parry-Smith W, Panikkar J. Risk factors for treatment failure following cold coagulation cervical treatment for CIN pathology: a cohort-based study. Arch Gynecol Obstet. 2015; 292(6):1329-1337.

23. Lima MI, Tafuri A, Araujo AC, de Miranda Lima L, Melo VH. Cervical intraepithelial neoplasia recurrence after conization in HIV-positive and HIV-negative women. Int J Gynaecol Obstet. 2009;104(2):100-104.
International Journal of Women's Health

\section{Publish your work in this journal}

The International Journal of Women's Health is an international, peerreviewed open-access journal publishing original research, reports, editorials, reviews and commentaries on all aspects of women's healthcare including gynecology, obstetrics, and breast cancer. The manuscript management system is completely online and includes

\section{Dovepress}

a very quick and fair peer-review system, which is all easy to use. Visit http://www.dovepress.com/testimonials.php to read real quotes from published authors. 